\title{
Covariance matrices for cross sections issued from optical model calculations
}

\author{
P. Dossantos-Uzarralde ${ }^{1}$, P. Mounier ${ }^{2}$, M. Brassart ${ }^{2}$, and M. Couvert ${ }^{3}$ \\ 1 CEA/DIF BP. 12, 91680 Bruyères-le-Châtel, France \\ 2 CEA/Saclay DM2S/SERMA, 91191 Gif-sur-Yvette, France \\ 3 CEA/Cadarache DTAP/SET, Bât. 220, 13108 Saint-Paul-lez-Durance, France
}

\begin{abstract}
Our interest is particularly focused at the study of uncertainties propagation within nuclear models. In this paper, a comparison between the most convenient approaches for generating covariance matrices for evaluations based on nuclear modeling calculations is presented. The first method involves the conventional matrix error propagation approach based on sensitivity matrix calculation. In the second one, uncertainties associated to the model parameters are propagated with a Monte Carlo simulation. To perform a quantitative uncertainty analysis, probability distributions must be assigned to each of the varied parameters, Monte Carlo procedures are used to simulate alternative distributions of parameter values.
\end{abstract}

\section{Introduction}

Nuclear reaction models play an important role in todays nuclear data evaluations. There are, however, difficulties in associating nuclear data evaluations with their uncertainties. In this general context, we discuss two distinct ways of calculating the nuclear cross section variance-covariance matrices issued from the spherical optical model. The use of these two methods is not a new idea, here, we attempt to evaluate the differences between the uncertainties generated by these methods. We first consider how data errors propagate through model calculations to affect error limits (or maximum error) of results. It's easy to first consider uncertainties for parameters used in the model calculations and then propagate these uncertainties to produce covariance informations for the derived results. The most convenient approaches is the direct deterministic calculation of uncertainty by calculation of sensitivity matrices which are multiplied by the covariance matrix of model parameters. Another method, which also needs the model parameter covariance matrix, propagates these uncertainties to the derived cross section by means of a Monte Carlo technique. This paper analyzes and compares the properties of both the classical sensitivity method and the Monte Carlo technique. Discussing simulation results for the calculation of all major neutron reactions on ${ }^{89} \mathrm{Y}$, we focused on the probability distribution function used for the Monte Carlo technique of quantifying covariance matrices. In particular we discuss how the parameter multivariate probability function distribution is applied to the problem of propagating errors. We also discuss the use of statistical regressions on values of the parameters to determine the relative importance of parameters contributing to the final uncertainty.

\section{The optical model potential}

The phenomenological optical model potential is defined [1] as follows:

$$
\begin{aligned}
\mathcal{U}(r, E)= & -\left[\mathcal{V}_{v}(r, E)+i \mathcal{W}_{v}(r, E)\right]-\left[\mathcal{V}_{s}(r, E)+i \mathcal{W}_{s}(r, E)\right] \\
& +\left[\mathcal{V}_{s o}(r, E)+i \mathcal{W}_{s o}(r, E)\right]+\mathcal{V}_{c}(r)
\end{aligned}
$$

The first 6 terms are factorized as the product of an energydependent potential depth and a radial-dependent geometrical form factor. The real potential, the volume and surface absorptions and the real and imaginary spin-orbit potentials are $\left\{V_{v}, V_{s}\right\}, W_{v}, W_{s}, V_{s o}, W_{s o}$, respectively. The radial form factors $f\left(r, a_{i}, R_{i}\right)$ are Woods-Saxon types with $R_{i}=r_{i} A^{1 / 3}$ where $r_{i}$ is the reduced radius. The depth $V$ of each of the six potential wells, the reduced radii $r_{i}$ and the diffusiveness $a_{i}$ may have an energy dependence. $r_{i}, a_{i}$ and $V$ are the parameters of the optical model. Consistent sets of parameters are used to calculate physical quantities such as total $\left(\sigma_{T}\right)$, shape elastic $\left(\sigma_{E}\right)$ and reaction $\left(\sigma_{R}\right)$ cross sections. Thus, the cross sections calculated with the spherical optical model can be characterized as a function of the model parameters:

$$
\begin{aligned}
\sigma_{j}= & f_{j}\left(V_{v}, V_{s}, V_{s o} ; W_{v}, W_{s}, W_{s o} ; a_{v_{s}}, a_{v_{s o}} ;\right. \\
& \left.a_{w_{v}}, a_{w_{s}}, a_{w_{s o}} ; r_{v_{v}}, r_{v_{s}}, r_{v_{s o}} ; r_{w_{v}}, r_{w_{s}}, r_{w_{s o}}\right) .
\end{aligned}
$$

The calculated system response $\mathbf{Y}_{j}=\sigma_{j, j \in\{T, E, R\}}$ is considered to be a real function of $p$ parameters, denoted $X_{1}, X_{2}, \ldots, X_{p}$. The response is related to the parameters via the computational model.

\section{Mathematical formalism}

\subsection{Deterministic approach}

First order Taylor's development. The classical model of prediction of errors is based on the propagation of the covariance of an input random perturbation. The dependence between the covariance matrices of the input and output can be determined using Taylor expansion (around the mean value). The Taylor series method is to approximate $f_{j}$ by a linear function. The linearizing greatly simplifies the error analysis at the expense of introducing an approximation.

$$
Y_{j} \approx f_{j}\left(\mu_{1}^{X}, \ldots, \mu_{p}^{X}\right)+\sum_{i=1}^{p}\left(\frac{\partial f_{j}}{\partial X_{i}}\left(\mu_{1}^{X}, \ldots, \mu_{p}^{X}\right)\right)\left(X_{i}-\mu_{i}^{X}\right)
$$


Mean value, variance, covariance:

$$
\begin{aligned}
\mu_{j}^{Y} & \approx f_{j}\left(\mu_{1}^{X}, \ldots, \mu_{p}^{X}\right) \\
\sigma_{j j}^{Y} & \approx E\left[\left(Y_{j}-\mu_{j}^{Y}\right)^{2}\right] \approx \sum_{i} \sum_{i^{\prime}} \frac{\partial f_{j}}{\partial X_{i}} \frac{\partial f_{j}}{\partial X_{i^{\prime}}} \sigma_{i i^{\prime}}^{X} \\
\sigma_{j j^{\prime}}^{Y} & \approx \sum_{i} \sum_{i^{\prime}} \frac{\partial f_{j}}{\partial X_{i}} \frac{\partial f_{j^{\prime}}}{\partial X_{i^{\prime}}} \sigma_{i i^{\prime}}^{X} .
\end{aligned}
$$

The results above is often written in matrix form as:

$$
\underline{\underline{V_{Y}}}=\underline{\underline{F_{X}}} \underline{\underline{V_{X}}} \underline{\underline{F_{X}}}
$$

where $T$ denotes the transposition, $\underline{V_{X}}$ the parameter covariance matrix and $F_{X}$ the sensitivity matrix.

In order to $\overline{\overline{\text { deal }}}$ with the nonlinear model (2) and the expansion to the second order of Taylor's development, the commonly employed uniform distribution $\mathcal{U}\left(\alpha_{i}, \beta_{i}\right)$ is used for the $V_{X}$ calculation. It should be noted that we have assumed the differentiability of $f_{j}$, which has not been proved mathematically. The sensitivities are then estimated by using a centered finite difference approximation.

Second order Taylor's development. The first order Taylor's expansion is usually employed to obtain an approximate estimation for the output error or the error bars. This technique is reliable and reasonable only if several stringent and severe assumptions are satisfied, such that the higher order error terms can safely be omitted in the expansion procedure. The goal of this paragraph is to estimate how the second order affects the results:

$$
\begin{aligned}
Y_{j} \approx & f_{j}\left(\mu_{1}^{X}, \ldots, \mu_{p}^{X}\right)+\sum_{i=1}^{p}\left(\frac{\partial f_{j}}{\partial X_{i}}\left(\mu_{1}^{X}, \ldots, \mu_{p}^{X}\right)\right)\left(X_{i}-\mu_{i}^{X}\right) \\
& +\frac{1}{2} \sum_{i=1}^{p} \sum_{i^{\prime}=1}^{p}\left(\frac{\partial^{2} f_{j}}{\partial X_{i} \partial X_{i^{\prime}}}\left(\mu_{1}^{X}, \ldots, \mu_{p}^{X}\right)\right)\left(X_{i}-\mu_{i}^{X}\right)\left(X_{i^{\prime}}-\mu_{i^{\prime}}^{X}\right) .
\end{aligned}
$$

Mean value, covariance:

$$
\mu_{j}^{Y}=f_{j}\left(\mu_{1}^{X}, \ldots, \mu_{p}^{X}\right)+\frac{1}{2} \sum_{i} \sum_{i^{\prime}} \frac{\partial^{2} f_{j}}{\partial X_{i} \partial X_{i^{\prime}}} \sigma_{i i^{\prime}}^{X}
$$

$$
\begin{aligned}
\sigma_{j j^{\prime}}^{Y}= & \sum_{i} \sum_{q} \frac{\partial f_{j}}{\partial X_{i}} \frac{\partial f_{j^{\prime}}}{\partial X_{q}} \sigma_{i q}^{X} \\
+ & \frac{1}{2} \sum_{i} \sum_{q} \sum_{q^{\prime}}\left(\frac{\partial f_{j}}{\partial X_{i}} \frac{\partial^{2} f_{j^{\prime}}}{\partial X_{q} \partial X_{q^{\prime}}}+\frac{\partial f_{j^{\prime}}}{\partial X_{i}} \frac{\partial^{2} f_{j}}{\partial X_{q} \partial X_{q^{\prime}}}\right) \\
& E\left[\left(X_{i}-\mu_{i}^{X}\right)\left(X_{q}-\mu_{q}^{X}\right)\left(X_{q^{\prime}}-\mu_{q^{\prime}}^{X}\right)\right] \\
+ & \frac{1}{4} \sum_{i} \sum_{i^{\prime}} \sum_{q} \sum_{q^{\prime}} \frac{\partial^{2} f_{j}}{\partial X_{i} \partial X_{i^{\prime}}} \frac{\partial^{2} f_{j^{\prime}}}{\partial X_{q} \partial X_{q^{\prime}}} \\
& \left(E\left[\left(X_{i}-\mu_{i}^{X}\right)\left(X_{i^{\prime}}-\mu_{i^{\prime}}^{X}\right)\left(X_{q}-\mu_{q}^{X}\right)\left(X_{q^{\prime}}-\mu_{q^{\prime}}^{X}\right)\right]-\sigma_{i i^{\prime}}^{X} \sigma_{q q^{\prime}}^{X}\right)
\end{aligned}
$$

Under the assumptions of independent parameters and continuous uniform distribution, we determine expressions for the elements of covariance matrix. In the calculation of the covariances, not only the covariances $\sigma_{i q}^{X}$ but also the fourth order moment is needed.

Final covariance $\operatorname{Cov}\left(Y_{j}, Y_{j^{\prime}}\right)=\sigma_{j j^{\prime}}^{Y}$ can be written:

$$
\begin{aligned}
\sigma_{j j^{\prime}}^{Y} & =\sum_{i=1}^{p} \frac{\partial f_{j}}{\partial X_{i}} \frac{\partial f_{j^{\prime}}}{\partial X_{i}} \sigma_{i i}^{X} \\
& +\frac{1}{4} \sum_{i=1}^{p} \frac{\partial^{2} f_{j}}{\partial X_{i}^{2}} \frac{\partial^{2} f_{j^{\prime}}}{\partial X_{i}^{2}}\left(E\left[\left(X_{i}-\mu_{i}^{X}\right)^{4}\right]-\left(\sigma_{i i}^{X}\right)^{2}\right)
\end{aligned}
$$

Introducing the second derivatives and the $4^{\text {th }}$ central moment diagonal matrix:

$$
\begin{gathered}
\underline{\underline{F_{X}^{2}}}=\left(\frac{\partial^{2} f_{j}}{\partial X_{i}^{2}}\right)_{\substack{1 \leq j \leq g \\
1 \leq i \leq p}} \\
\underline{\underline{Q_{X}}}=\left(Q_{X i j}\right)_{\substack{1 \leq i \leq p \\
1 \leq j \leq p}}=\left\{\begin{array}{ll}
0 & \text { si } i \neq j \\
E\left[\left(X_{i}-\mu_{i}^{X}\right)^{4}\right] & \text { si } i=j
\end{array} .\right.
\end{gathered}
$$

The results above can be written in matrix form as:

$$
\underline{\underline{V_{Y}}}=\underline{\underline{F_{X}}} \underline{\underline{V_{X}}} \underline{\underline{F_{X}}}+\frac{1}{4} \underline{\underline{F_{X}^{2}}}\left(\underline{\underline{Q_{X}}}-\underline{\underline{V_{X}^{2}}}\right) \underline{\underline{F_{X}^{2}}}
$$

where $V_{X}$ here denotes the diagonal covariance matrix and $F_{X}$ the sensitivity matrix.

Using the uniform distribution $U\left(\alpha_{i}, \beta_{i}\right)$ :

$$
\begin{aligned}
E\left[\left(X_{i}-\mu_{i}^{X}\right)^{4}\right] & =\frac{\left(\beta_{i}-\alpha_{i}\right)^{4}}{80} \\
& =\frac{\left(\alpha_{X_{i}}\left|\mu_{i}^{X}\right|\right)^{4}}{5}\left\{\begin{array}{l}
\mu_{i}^{X}=\frac{\alpha_{i}+\beta_{i}}{2} \\
\alpha_{i}=\mu_{i}^{X}-\alpha_{X_{i}}\left|\mu_{i}^{X}\right| \\
\beta_{i}=\mu_{i}^{X}+\alpha_{X_{i}} \mid \mu_{i}^{X}
\end{array}\right.
\end{aligned}
$$

In an analogous way one can obtain finite difference approximations to higher order of the derivatives and differential operators. We use the three point central difference approximation to obtain the second derivatives of $f_{j}$.

\subsection{Monte Carlo method}

The goal of the Monte Carlo method is to determine how random variation of the parameter values affects the sensitivity of the system that is being modeled. Monte Carlo simulation is categorized as a sampling method because the inputs are

(8) randomly generated from probability distributions. So, we try to choose a distribution for the inputs that most closely matches parameters values we already have.

Uniform distribution. In the context of a comparison between both methods, we used the uniform distribution $\mathcal{U}\left(\alpha_{i}, \beta_{i}\right)$ as parameter probability function distribution (pdf) to be coherent with the sensitivity approach. The pdf of the parameter vector $X$ is the product of the marginal distributions $f_{X_{i}}$ of each parameters $X_{i}: f_{\underline{X}}=\prod_{i=1}^{p} f_{X_{i}}$. 


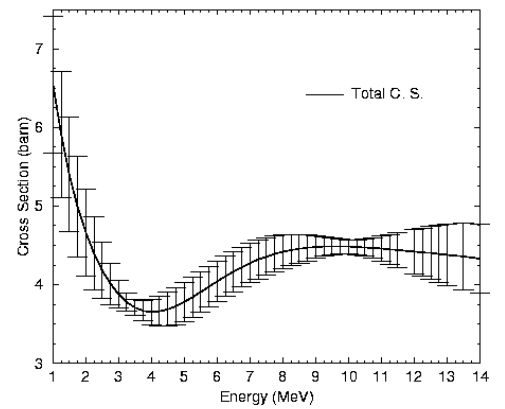

Fig. 1. Mean total cross section and relative error calculated by the Monte Carlo method.

Multivariate normal distribution. We now turn to explore the multi-variate pdf, the most probable distribution of the unknown theoretical parameter distribution, justified by the Shannon's theory of information [3]. The functional form of this pdf is:

$$
f_{\underline{X}}(\underline{X})=\frac{1}{(2 \pi)^{p / 2}\left|\underline{\underline{V_{X}}}\right|^{1 / 2}} \exp \left(-\frac{1}{2}(\underline{X}-\underline{\mu})^{T}{\underline{\underline{V_{X}}}}^{-1}(\underline{X}-\underline{\mu})\right)
$$

where the -1 denotes the inverse of the matrix, $\underline{V_{X}}$ denotes the parameter variance covariance matrix, $\left|\underline{V_{X}}\right| \overline{\overline{\text { denotes }}}$ the determinant of $\underline{V_{X}}, \underline{X}$ is the vector of $X_{p}$ values, and $\underline{\mu}$ is the vector of the means of the $p$ distributions. $\underline{\underline{V_{X}}}$ is symmetric and positive definite. Because of this, the covariance matrix of the estimated parameters can be calculated by :

$$
\underline{\underline{V_{X}}}=\underline{\underline{P}} \underline{\underline{D}} \underline{\underline{P}}^{T}
$$

where $\underline{\underline{P}}$ is the orthogonal matrix whose columns are the eigenvectors and $\underline{D}$ the diagonal matrix of eigenvalues. The random vector $\tau \overline{\overline{\text { can }}}$ be derived by the method of Box and Muller [4] from a sequence of uniform deviates such that $\tau$ corresponds to independent random variables drawn from the standard normal distribution $\mathrm{N}(0, \mathrm{I})$, having a mean vector of 0 and covariance matrix equal to the identity matrix $I$. Thus, once the matrix $\underline{P}$ has been derived from the covariance matrix $\underline{V_{X}}$, repeated application of equation $\underline{X}=\underline{\underline{P}} \underline{\underline{D}} \underline{\tau}+\underline{\mu}$ to successive random vectors $\tau$ yields a large sampling of independent random data vectors, each vector corresponding to an independent observation of the variables from the specified multivariate normal distribution. The effect of parameter uncertainty on a model with adjusted parameters must be taken into account for the accurate estimation of the output error. Figure 1 plots the mean total cross section and error bars calculated by the Monte Carlo method.

\section{Results}

$\sigma$ symbolizes the derived physical quantities generated by optical model calculations. Calculations have been performed for

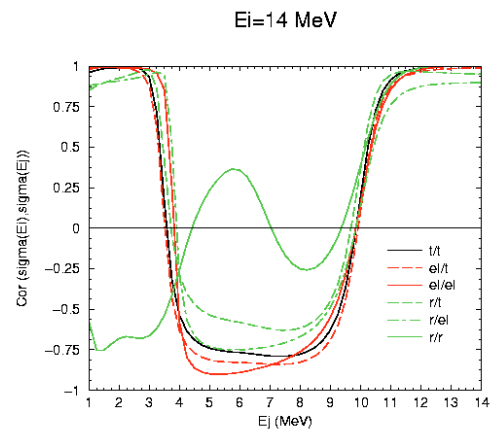

Fig. 2. $n+{ }^{89} \mathrm{Y} 14 \mathrm{MeV}$ cross section correlations obtained by the first order Taylor's expansion.

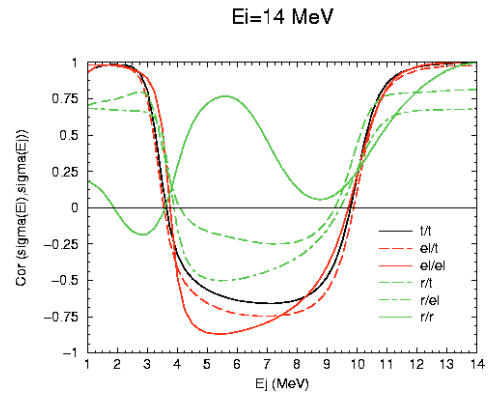

Fig. 3. $n+{ }^{89} \mathrm{Y} 14 \mathrm{MeV}$ cross section correlations obtained by the Monte Carlo method.

all the cross sections with the code SCAT2000 [1], [2]. Figures 2 and 3 show the evolution of the cross section $\xi \xi^{\prime}$ correlations $\operatorname{Cor}\left(\sigma_{\xi}\left(E_{i}\right), \sigma_{\xi^{\prime}}\left(E_{j}\right)\right),\left(\left(\xi, \xi^{\prime}\right) \in\{\right.$ total, elastic, reaction $\} \times$ \{total, elastic, reaction\}) obtained respectively by the sensitivity method (fig. 2) and the Monte Carlo method (fig. 3). These results are summarized on figure 4 . With the second order Taylor's development (fig. 5), a better agreement is seen between the two methods.

\section{Correlations of parameters uncertainties}

In order to estimate the uncertainty of the target output using equation (13), one needs an algorithm to estimate the uncertainty of parameters $V_{X}$. To obtain $V_{X}$, we have to find parameter values that minimize the difference between the observed experimental observations and the chosen model. This difference is assumed to be due to errors in the experimental determination of the data points, and thus it is common to describe the entire model-fitting process in terms of minimization of an error function. The most common representation for regression models is based on the chi-square distribution and its associated statistic $\chi^{2}$. This nonlinear least squares problem can be solved with Levenberg-Marquardt minimization algorithm [5]. However, it must be initialized by estimates around the solution for unknown parameters. Figure 6 shows an example of optimization using a set of parameters initialized with the Koning-Delaroche parameter evaluation [6]. Experimental data are from EXFOR [7].

Given standard results for the $V_{X}$, matrices should be estimated in a robust way. Here, the Principal Component 


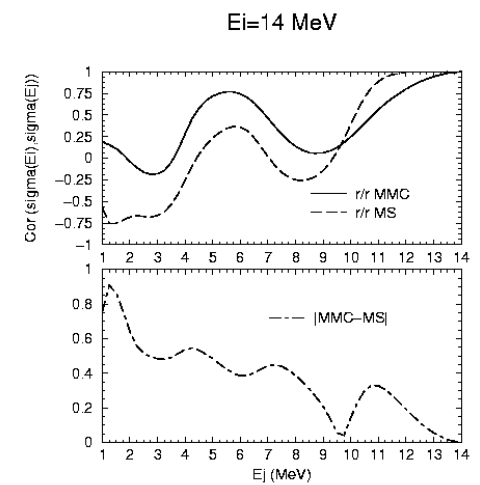

Fig. 4. Comparison of the reaction cross section correlation calculated by the Monte Carlo method (MMC) and by the sensitivity method (first order Taylor's development) (MS). Upper panel: comparisons of correlations. Lower panel: correlation absolute differences.

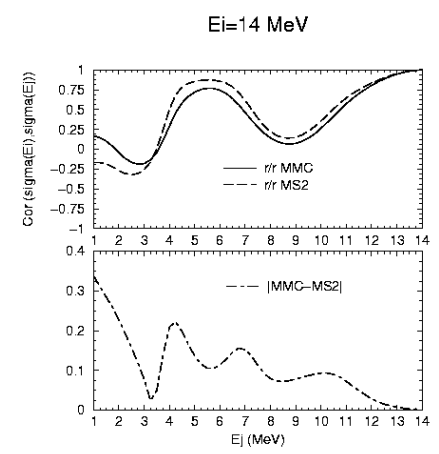

Fig. 5. Comparison of the reaction cross section correlation calculated by the Monte Carlo method (MMC) and by the sensitivity method (second order Taylor's development) (MS). Upper panel: comparisons of correlations. Lower panel: correlation absolute differences.

Analysis technique, has been used to detect linear parameter combinations mainly by reducing their number to find the dominant ones (fig. 7).

\section{Conclusion}

The objective of the present paper is to report on an investigation of the use of error propagation techniques for the estimation of covariance matrices for nuclear cross sections by optical model calculations. The main shortcomming of the sensitivity method is that the results are approximate only. Moreover, it may not always be easy to determine whether the approximations involved using this method are acceptable. The Monte Carlo method does not suffer from this problem, because it can reach an arbitrary level of accuracy. The Monte Carlo method will probably also be preferred when error propagation with a complex nuclear reaction code (TALYS [8]) is studied, because the method is more easily implemented and generally applicable. The main difficulty with the Monte Carlo method (which is also shared by the sensitvity method) is that error propagation can only be performed once the covariance matrix of the model parameters is available.

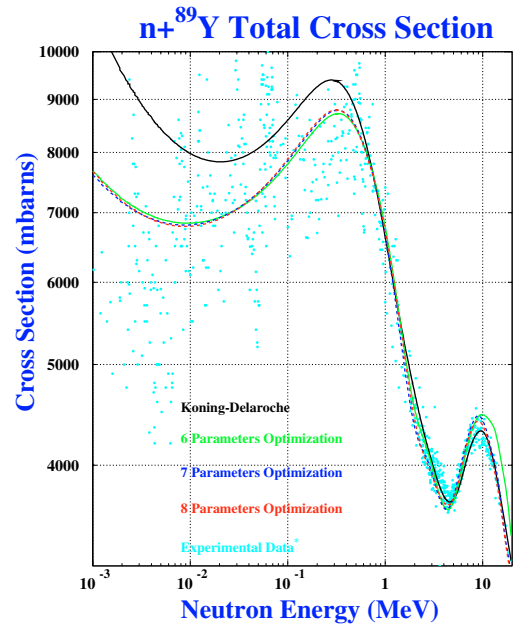

Fig. 6. $n+{ }^{89} Y$ total cross section - Parameters optimization obtained with Levenberg-Marquardt minimization algorithm.

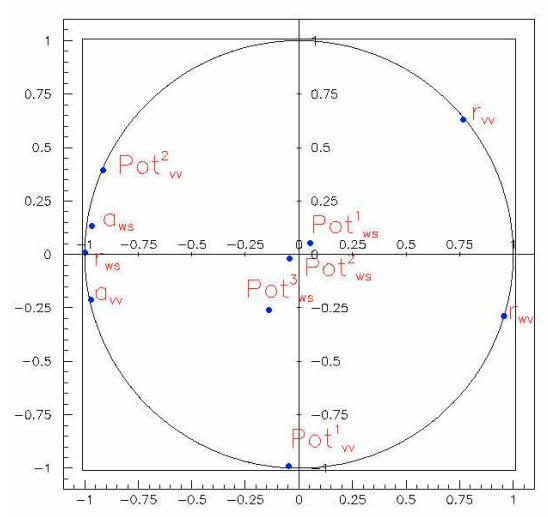

Fig. 7. Example of Principal Component Analysis used for a 10 parameter optimization.

\section{References}

1. O. Bersillon, SCAT2: Un programme de modèle optique sphérique, CEA-N-2227, NEANDC(E), 220 L, INDC(FR) 49/L, 1981.

2. O. Bersillon, The Computer Code SCAT2, Workshop on Applied Nuclear Theory and Nuclear Model Calculations for Nuclear Technology Applications, edited by M.K. Mehta, J.J. Schmidt, 1988.

3. F.H. Fröhner, Évaluation et analyse des données relatives aux résonances nucléaires, rapport JEFF-18, OCDE, Agence pour l'Énergie Nucléaire.

4. G.E. Box, M.E. Muller, A note on the generation of random normal deviates, Annals Math. Stat, 29, (1958), pp. 610-611.

5. D.W. Marquardt, Journal of the Society for Industrial and Applied Mathematics 11, pp. 437-441.

6. J.-P. Delaroche, A.J. Koning, Local and global nucleon optical models from $1 \mathrm{keV}$ to $200 \mathrm{MeV}$, Nucl. Phys. A 713, 231 (2003).

7. Base de données expérimentales EXFOR, http://www.nea.fr/html/dbdata/data/experimental.htm.

8. A.J. Koning, S. Hilaire, M. Duijvestijn (these proceedings). 